\title{
Performance Evaluation of a Protocol for Fair P2P Auctions over MANETs
}

\author{
Ines Doghri and Hella Kaffel-Ben Ayed
}

\begin{abstract}
In this paper, we explore the deployment of P2P auctions over MANETs. We define a $\mathrm{P} 2 \mathrm{P}$ auction process and identify the need to have a fair registration and a fair bidding. We propose a distributed communication architecture and a protocol to support this kind of auctions. We perform a formal specification and verification of the BSAP protocol using PROMELA/SPIN and evaluate its performance.
\end{abstract}

\section{Introduction}

With the deployment of wireless communication and mobile computing, new ways for people to interact with each other and their surrounding environment are emerging. Mobile ad hoc networks (MANETs) do not need any infrastructure or management entity. They are characterised by their completely autonomous, dynamic, auto-organised and ubiquitous nature. The most attractive economic benefit is the low cost of this communicating infrastructure and of its management.

At the same time, peer-to-peer (P2P) systems constitute nowadays an increasingly important part of the online world. Ad hoc networks present similarities with P2P systems in terms of decentralization, equality and autonomy. This will help the raise of new P2P applications over MANETs such as mobile commerce. We propose here a novel concept: P2P auctions over MANETs.

In this paper, we first present the motivation and business scenario of this work. Then, we propose an architecture and a communication protocol to support P2P auctions over MANETS. The formal specification and verification of this protocol using PROMELA and SPIN are presented. Performance evaluation results are discussed. Afterwards, we discuss related work. Finally, we present the concluding remarks and the ongoing work.

Ines Doghri*, Hella Kaffel-Ben Ayed**

National School of Computer Science, CRISTAL Laboratory, University of Manouba, Tunisia, e-mail: *ines.doghri@gmail.com, **Hella.kaffel@fst.rnu.tn

Please use the following format when citing this chapter

Doghri, I., H. Kallel-Ben Ayed, 2008, in IFIP International Federation for Inlormation Processing, Volume 265, Advances in $A d$ Hoc Networking, eds. Cuenca, P., Guerrero C., Puigjaner, R., Serra, B., (Boston: Springer), pp. 85-96. 


\section{Motivation and problematic}

During the last years, auctions became the major phenomenon of electronic commerce and are widely used to sell a variety of commodities, such as treasury bills, mineral rights, art works, etc. An auction is usually defined as a market institution with an explicit set of rules determining resource allocation and prices on the basis of bids from the market participants [1]. The commonly seen auctions types include English auction, Dutch auction and Vickrey auction [2]. New mobile and wireless networks types emerged, offering novel and interesting capabilities. They offer new ways of interactions. Users are nowadays equipped with sophisticated wireless terminals in order to be always connected and therefore to consult their e-mails, to work remotely with their companies, or to make e-commerce transactions. The ETSI (European Telecommunications Standards Institute) has defined the mobile commerce as: "The efficient delivery of electronic commerce capabilities, directly in the consumer's hand anywhere, anytime, via wireless technology" [10].

In the present study, we foresee ad hoc networks as a support for m-commerce and particularly for auctions. An auction process could be created anywhere as soon as a group of at least three persons equipped with wireless terminals agree to participate in the auction. One of them announces goods for sale or for purchase. The others would play the role of competing bidders that would submit bids. We could imagine various scenarios of spontaneous markets created temporarily for an auction event; such as markets set up in a harbour for the sale of stocks of fish or within the framework of a farm or in airport for the sales of last minute plane tickets. Nomadic exhibitions may also raise spontaneous $\mathrm{P} 2 \mathrm{P}$ auction events. This way of deploying auctions may also be considered as an extension of auction events occurring over an infrastructure network, permitting this way to MANET users to participate to such markets. These scenarios imply multiple advantages and motivations such as ubiquity, convenience, availability, affordability and opportunity. However, the deployment of auction over MANETs raises various problems (security, trust, robustness, fairness, etc) resulting from the nature of these networks.

\section{WAHS: a full distributed auction architecture}

\subsection{The architecture}

Most Internet based auctions model usually rely on a central auction server (the auctioneer). This latter performs various functionalities and can implement multiple auction-related policies. The model of $\mathrm{P} 2 \mathrm{P}$ auction over ad hoc networks [4] bears a resemblance to P2P networking model of ad hoc networks. Hence, we propose new communication architecture, called WAHS (Wireless Auction Handling System), to support $\mathrm{P} 2 \mathrm{P}$ auctions over mobile ad hoc networks. It is based on the distribution of 
the auction site to fit the ad hoc context, but also to ensure a high level of security. Several studies removed the auctioneer from their auction models [8], [6] and [9]. All these works proved that omitting the auctioneer is due to autonomy considerations. Furthermore, in our context, decentralization prevents the auction inhibition in the case where the auctioneer becomes non-connected to the network.

We define one functional component that supports all the auctioneer functionalities', named BSAP (Buyer/Seller Agent Peer). It is associated with an auction initiator as well as with each bidder participating to the auction. One protocol, i.e. BSA Peer protocol (BSAP-protocol), is defined to support interactions between the functional components (the peers) of WAHS. P2P auctions over MANETs present similarities with the real time auctions. The following assumptions can be made about this type of markets: (i) These auctions would require the physical presence of bidders or their mobile devices since the beginning of the auction, (ii) The probability of new arrivals during the process is low, (iii) the mobility of mobile nodes is low (about $1 \mathrm{~m} / \mathrm{sec}$ ), (iv) the nodes moving zone depends on the place where the market is set up (port, farm, airport, market, etc.) and (v) auctions are limited in duration.

\subsection{The BSAP protocol specification}

\subsubsection{The P2P auction process}

For the sake of simplicity we consider English auctions as a case study in the rest of the paper. However, the proposed architecture and protocol can be used to model any kind of open cry auction protocol. We devide the P2P auction process into three stages:

\section{Stage 1. The Initialization phase}

1-Auction advertisement: The initiator, a peer connected to the ad hoc networks who has a good to sell, initiates the auction application by broadcasting an "auction_advertise" message in order to inform all nodes about the auction event to be set up.

2- Auction access: Each interested node sends a "register_bidder" message to the initiator as soon as he receives the "auction_advertise" message. This latter becomes a registered participant of this auction.

3-Auction creation: After collecting all "register_bidder" messages, the initiator sets its primary list of members. He sends to all registered peers an "auction_create" message (in multicast) containing the list of participants (Access Control List). The ACL will allow further access control to the auction. The choice of multicast is in the purpose to satisfy the need for communication of group and to do not reveal the information of bidding to unrecorded nodes.

\section{Stage 2. The Bidding phase}

4- Submission: The first round begins for each bidder as soon as he receives the "auction_create" message. It sends its bid in a "submit_bid" message to all the members of the group and collects the others bids. Before accepting a bid, every bidder 
verifies if the sender is in the list; if not, it rejects its bid. Each peer computes the best bid and decides if it is going to outbid in the next round.

5- Auction quit: A bidder can leave at any time an auction by sending an "auction_exit" message to all members within the ACL.

6- New Auction access during the bidding phase: If a node $\mathrm{J}$ within the ad hoc network wants to access an ongoing auction, it starts a neighbour discovery process using the expanded ring search (ERS) technique [13]. Auction REQ message is used for this purpose. When a member of the auction I receives an Auction REQ message from node $J$, it sends back an Auction REP message to J, so that node $J$ will record the address of the auction initiator and the node $J$ will stop the neighbour discovery process. After that, node $J$ contacts the initiator to join the auction process. If the initiator sends back to node $J$ his acceptance, then $\mathbf{J}$ does registration. After doing all the necessary update, the initiator sends an auction_create message to all the list members.(cf. Fig. 1)

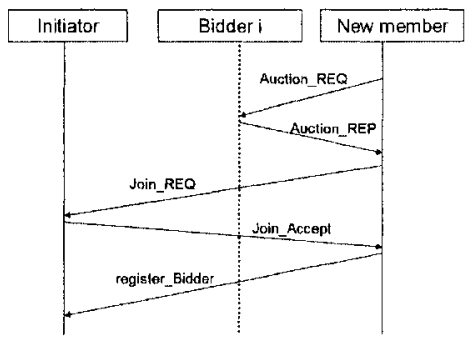

Fig. 1 Joining an auction process

\section{Stage 3. Closing phase}

At the end of the auction life, every bidder who believes that he submitted the highest bid during the very last round, sends a "winner_notif" message to the initiator. This avoid a possible inconsistency when many winners exist with different prices resulting to loss "submit_bid" messages due to wireless communication or congestion, etc. The initiator collects all "winner_notif" messages and compares the prices to identify the best bid. The transaction settlement occurs between the initiator and the winner.

\subsubsection{The BSAP formal Specification \& Verification}

\section{a. Modeling a broadcast system under SPIN}

We used the PROMELA language to specify the BSAP protocol and SPIN to verify it. These tools are usually used to specify point to point communication. When two nodes want to communicate they use one predetermined channel which makes the connection between them [11]. Therefore, we implemented some add-ins under PROMELA to adapt SPIN to the modeling and the checking of protocols operat- 
ing over Ad-hoc network. To model the CSMA/CA (Collision Avoidance) mode of transmission, we defined only one channel of communication which is indexed according to the number of nodes (this is also called matrix of the channels in [11]). Here is an example:

\#define $n 3 / *^{\text {nodes number*/ }}$

mtype $=$ auction_advertise, register_bidder,auction_create, submit_bid,winner_notif; $/{ }^{*} B S A P$ messages*/

Chan $q[n]=[1]$ of mtype, byte; $/ *$ indexed channel for broadcast $* /$

b. BSAP protocol Formal specification " automata "

We describe the different states of the BSAP bidder using the automata illustrated in the figure 2. In this figure, all circles represent the different states and transitions represent the events in/out from a state to another.

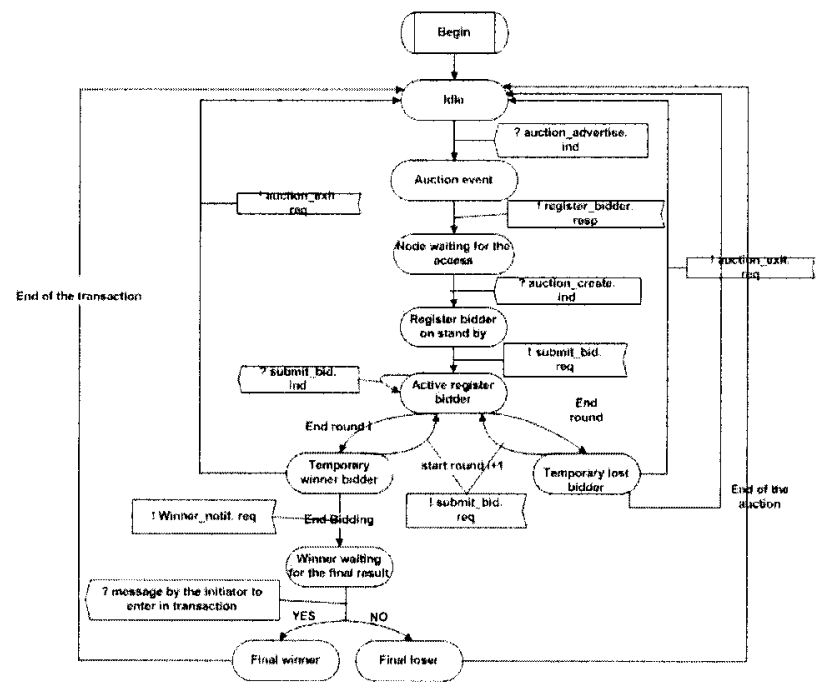

Fig. 2 The bidder automata

\section{c. BSAP protocol verification}

For the verification of the BSAP, we used the simulation facility of the validation tool SPIN to support early fault detection. Especially the message sequence chart (MSC) facility was very supportive in examining the communication pattern of our design. An important number of simulations has been made before finding a model which suits the requirements. A simulation of the basic BSAP Model is described on figure 3. Each line represents a process: BSAP initiator, BSAP bidder 1, etc. Each box represents one step in the process. The number in the box corresponds to the simulation step. Arrows correspond to the message transfer, and numbers on these arrows correspond to the transmitted message value. Also, "!" means sending and 
"?" means receiving as described in [11]. The verification parameters are checked with no errors (no invalid end-state, no deadlock and no loop).

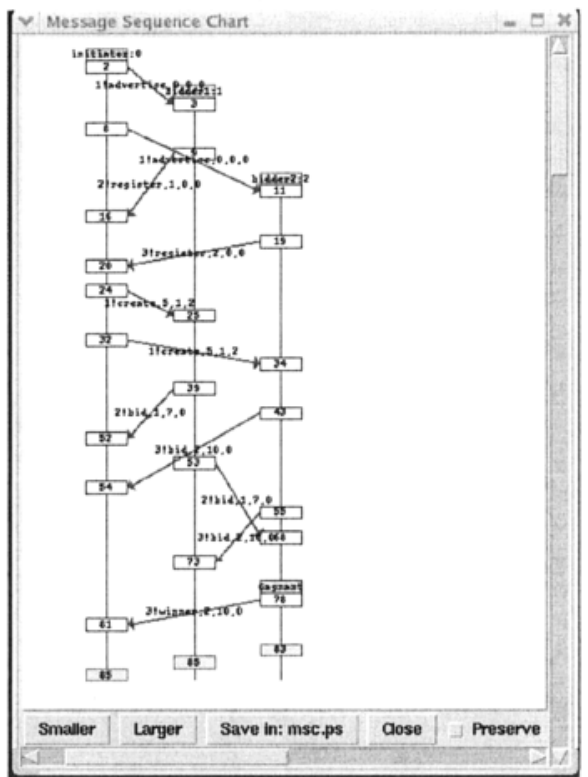

Fig. 3 Simulation of the basic BSAP-protocol model

\subsection{The fairness mechanism}

Fairness is a big challenge for auctions. It means that all participants should be treated equally [8]. It concerns essentially the bidding process and means that no bidder should have more information than any other to determine their bid, all submitted bids during a round should be evaluated during the same round and the bidder sending the highest bid should win the auction [12]. When auctions are deployed over ad hoc networks, intermediary bidders can receive bids and react before the other bidders and may finally win the auction. To prevent this, and provide a fair registration and a fair bidding, we assume that the initiator has to wait for a period of time, named Tregister, to collect registrations and each bidder has to wait for a period of time, named Tfair, to collect all bids. In the next section, we determine by simulation the estimations of Tfair and Tregister. 


\section{Performance evaluation}

The goals of simulations are to: (i) compare the values of Tfair obtained by simulation with those obtained by one related work (eq. 3) and noted Tfair/[4], (ii) evaluate Tfair and (iii) estimate the communication overhead.

\subsection{The simulation environment}

The simulations were performed using the network simulator NS-2 with CMU extensions to support MANETs. We used the random waypoint mobility model since it considered as the best model for MANETs [14]. The link layer was implemented using IEEE 802.11 Distributed Coordination Function (DCF) and Medium Access Control Protocol (MAC). Taking into account the assumptions on the context of our application as in section 2, we choose a mobility scenario characterized by: the speed of the nodes in the network is 1 meters/second and the pause time is 5 seconds. We used AODV as a routing protocol since it is available over NS2; although any other routing protocol can be used. In order to have the 95 percent confidence interval for the mean of each gathered static, we run 30 simulations for each scenario.

\subsection{The metrics}

- To determine Tregister: we define a metric named "Register-out". Tregister value is the value of optimal register timeout when "Register-out" (eq. 1) reaches zero. This metric is calculated as follows:

$$
\text { Register }- \text { out }=\frac{\text { register }-N b-o u t}{\operatorname{Reg} N b}
$$

With:

Register - out $=$ Rate of registrations out register timeout.

Register $-\mathrm{Nb}-$ out $=$ number of register_bidder messages received out register timeout.

$R e g N b=$ total number of all registrations received by the initiator.

- To determine Tfair: we define a metric named "Bids-out-rnd". Tfair value corresponds to the optimal round timeout when "Bids-out-rnd" (eq. 2) is null. This metric value is computed in the following manner: 


$$
\text { Bids }- \text { out }-r n d=\frac{\sum_{j=1 . . S i z e-A C L} \text { bids }- \text { out }-r n d / \text { bidder }_{j}}{\text { Size }-A C L}
$$

With:

bids - out $-r n d /$ bidder $=$ Rate of bids received per each bidder after the round timeout.

Size $-A C L=$ the number of registered members.

- To evaluate the overhead of our protocol by computing the number of unicast messages. This includes the various unicast messages required namely register_bidder, auction_create and submit_bid. This indicates the communication overhead incurred by the protocol.

\subsection{The simulation results}

We first evaluated the BSAP-Protocol. To determine the optimal values of Tregister and Tfair, we varied two simulation timers: (i) Register timeout marking the end of the waiting time of the initiator during the initialization phase and (ii) the round timeout marking the end of a round duration during the bidding phase.

a. Tfair/BSAP obtained by simulation vs theoretical Tfair/[4]

In order to compare the fair round duration induced by our protocol with Tfair/[4], we used the scenarios shown in the table 1. These scenarios consider MANETS with static topologies, fixed nodes and a coverage area of 50 meters. The last line in this table corresponds to the Tfair/[4] for each scenario.

\begin{tabular}{|c|c|c|c|}
\hline & Scenario 1 & Scenario 2 & Scenario 3 \\
\hline Network size & $500 \mathrm{~m}^{*} 500 \mathrm{~m}$ & $250 \mathrm{~m}^{*} 250 \mathrm{~m}$ & $50 \mathrm{~m}^{*} 50 \mathrm{~m}$ \\
\hline Maximal hops number & 14 & 7 & 1 \\
\hline Tfair & $12.6 \mathrm{~s}$ & $6.3 \mathrm{~s}$ & $0.9 \mathrm{~s}$ \\
\hline
\end{tabular}

Table 1 Simulation scenarios

Fig. 4 shows that the curve of Tfair/[4] diverges with that of Tfair/BSAP. We observe that Tfair/BSAP values increase by the rise of the number of hops but not linearly. The theoretical Tfair/[4] is then too large. Hence, we progress towards a dynamic Tfair. For that, the goal of the following simulations is to determine the factors affecting Tregister and Tfair.

b. Impact of varying node population on Tregister and Tfair

We varied the node population and analyzed by simulation the behaviour of the BSAP. This population is varied from 10 nodes to 100 nodes. The considered areas of the network were $183 \mathrm{~m} \times 183 \mathrm{~m}, 258 \mathrm{~m} \times 258 \mathrm{~m}, 408 \mathrm{mx} 408 \mathrm{~m}, 483 \mathrm{~m} \times 483 \mathrm{~m}$ and $578 \mathrm{~m} \times 578 \mathrm{~m}$ for the $10,20,50,70$ and 100 node networks, respectively (ensuring the network density of around 300 nodes $=\mathrm{km}^{2}$ ).

Fig. 5 , shows the rise of Tregister with the rise of nodes population. In order to 


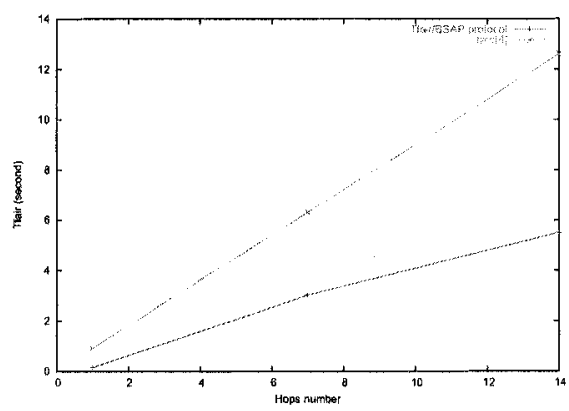

Fig. 4 Tfair vs hops number

have the maximum number of buyers, the initiator must wait for 3 seconds to allow up 10 bidders to join the auction and 20 seconds to have 100 bidders. In Fig. 6 , we see that all the curves are decreasing and the optimal round duration (Tfair) is varying with the number of nodes. In fact, the number of submit bid messages out of round reaches zero as follows: for 20 population node the mean Tfair is $6 \mathrm{sec}$; for 50 population node the mean Tfair is $25 \mathrm{sec}$; and for 70 population node the mean Tfair is $30 \mathrm{sec}$. For simulations in Fig. 6 , we used Tregister values from the Fig. 5 to have the maximum number of participants.

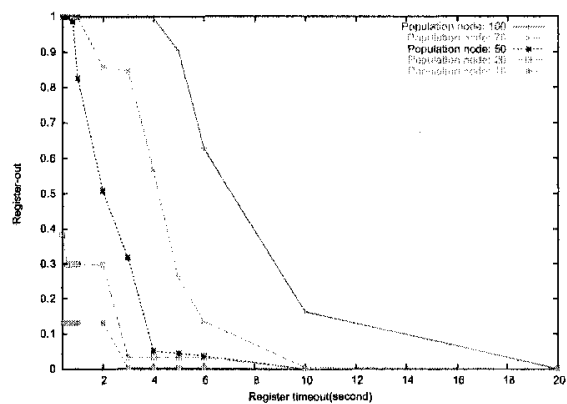

Fig. 5 Initialization phase: Register timeout versus Register-out Rate

\section{c. Impact of varying node population on number of unicast messages}

In Fig. 7, the total number of messages exchanged increases with the number of peer nodes. The Fig. 7 (a) shows that the number of create messages is the same as the number of register messages. That is logic because nodes who send their registration will receive the auction_create message from the initiator. But, when the number of peers increases to reach a high value as 100 nodes, the number of auction_create messages becomes lower than the number of register_bidder messages. This shows that there is some registration packets lost by the network. The use of 


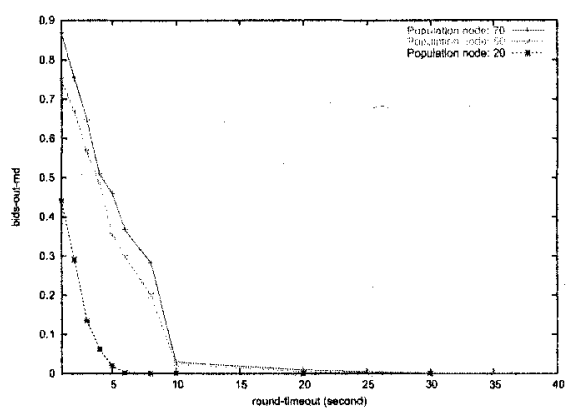

Fig. 6 Bidding phase: Round timeout versus Bids-out-rnd Rate

a reliable transport protocol would allow avoiding this loss. We can note from the Fig. 7 (b) that the curve has an exponential rise according to the increase of the node population. This result underlines our choice of the multicast communication mode in order to not overload the network by unicast messages. This is well adapted to satisfy the need of the many-to-many communication required by our BSAP protocol during the bidding process.

\section{Related work}

The work presented in [3] proposes the deployment of auctions over the mobile networks. The bidding information is disseminated in the network by the auctioneer and interested bidders submit their settings. Fairness is fulfilled by the setting of a "Timer Waiting". Bids are saved within the network layer of the auctioneer. When the timer expires, the auctioneer network layer delivers all the information received to the application for evaluation. The best bid is then sent to all participants. This approach presents many weaknesses such as: the periodic flooding of the current auction information in the network by the initiator that implies the messages redundancy. The trust in the auctioneer and the relaying of messages by intermediate nodes raises reliability and security problems. The presence of a central entity (i.e. the auctioneer) is not suitable in the context of ad hoc networks. At last, this approach introduces processing within the network layer.

In [5], the authors present a self-organizing distributed auction system using a mobile multihop ad-hoc network as a communication platform. In order to substantially increase the probability that negotiating peers successfully reach an agreement, communication is focused on a static geographic area, called the marketplace. To ensure a reliable communication channel between two devices, the proposed protocol assumes at least one copy of the agent exists. However, this solution may cause problems at the marketplace when agents make different agreements.

The work [4] aims at deploying a model of auction over an ad hoc network by pro- 

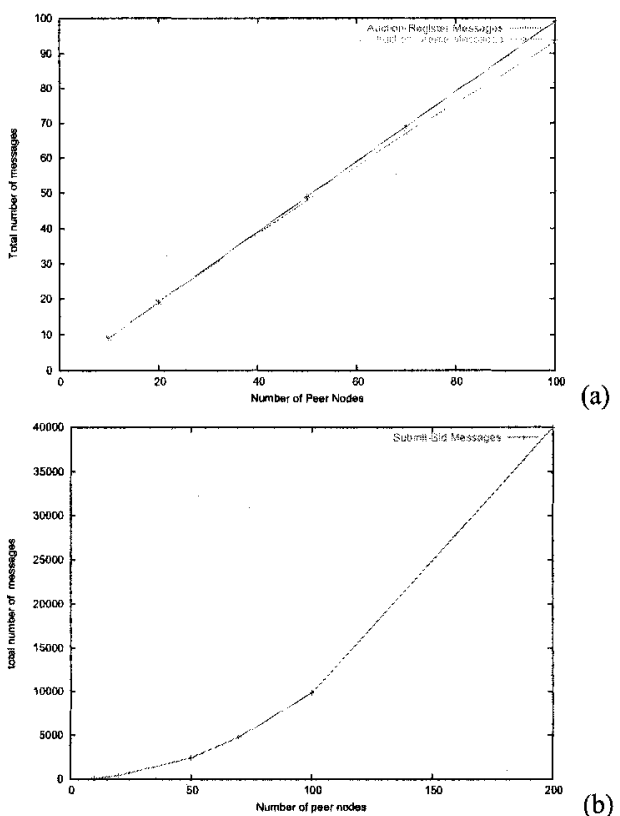

(b)

Fig. 7 Communication overhead for varying node population: Node population versus number of unicast messages both in (a) the initialization phase and (b) the bidding phase

viding fairness requirements. This model is based on the distribution of the auction site to fit the ad hoc context, but also to ensure a high level of security. Here, the authors proposed an estimation of Tfair:

$$
\text { T fair } /[4]=0.982656 * \text { MaximalHopsNumber }
$$

This solution design ensures the faimess but does not manage a dynamic number of participants, the nodes mobility. We note that to provide fairness, this approach requires the maximal hops number information. This latter information is provided by the network layer which creates a dependency of the approach on the network layer.

\section{Conclusion \& Future Work}

In this paper, we proposed a distributed architecture and a protocol to support $\mathrm{P} 2 \mathrm{P}$ auctions over MANETs. We focused on fairness and defined two time durations: Tregister and Tfair to provide a fair registration and a fair bidding. First, we im- 
plemented the broadcast system under SPIN model-checker and formally verified the BSAP protocol correctness. Then, comparison to one related work [4] show that Tfair has a slight increase in a linear manner according to the hops number. The simulation results show that Tfair depends on the number of nodes. Furthermore, we note that our protocol incurs low communication overhead. Compared to related works, our proposal has the advantage of being flexible with regards to both the MANETs' environment and the auction rules and technology. Ongoing work is on: 1) more simulation experiments by varying other network parameters, 2) assess the theoretical formula to estimate a dynamic Tfair and 3) on security of the BSAP protocol.

\section{References}

1. R.P.McAfee and J.McMillan, R.P.McAfee and J.McMillan . Auctions and bidding, Journal of Economic Literature, 25:699-738, 1987.

2. P.Klemperer,editor, The economic Theory of Auctions (2 vols.). Edward Elgar Publishing, 2000.

3. N.Lin, S.Shrivastava, "System support for small scale auctions", In proc. IFIP Med-Hoc-Net, Mahdia, Tunisia, June 2003.

4. A.Fourati, K.Al Agha, H.K. Ben Ayed "Secure and fair auctions over ad hoc networks", International Journal of Electronic Business(IJEB), Vol 5,pages:276 - 293, 2007.

5. H. Frey, D. Gorgen, J. K. Lehnert, P. Sturm, "Auctions in mobile multihop ad-hoc networks following the marketplace communication pattern",6th International Conference on Enterprise Information Systems ICEIS'04, Porto, Portugal, 2004.

6. B Rachlevsky-Reich, I Ben-Shaul, N T Chan, A Lo, and T Poggio, "GEM: A Global Electronic Market System" Information Systems Vol. 24, No. 6, pp. 495-518, 1999.

7. D.Hausheer, B.Stiller, "PeerMart : The Technology for a Distributed Auction-based Market for Peer-to-Peer Services", 2004. http://www.peermart,net/

8. Paul D. Ezhilchelvan and G. Morgan, "A Dependable Distributed Auction System: Architecture and an Implementation Framework", Fifth International Symposium on Autonomous Decentralized Systems (ISADS 2001), USA, March 26-28, 2001.

9. F. Brandt, "Secure and Private Auctions without Auctioneers", Technical Report FKI- 245-02, ISSN 0941-6358, Department of Computer sciences, technical university of Munich, February 2002 .

10. ETSI "Mobile Commerce, Mobile Signature Service, Security Framework", Technical Report, France, (2003)

11. R.Gerth, "A concise language reference for promela", http://spinroot.com/spin/Man/Quick.html.

12. N. Asokan, "Fairness in Electronic Commerce", Technical report, IBM Research Division, March 1997.

13. C.E. Perkins, E.M. Royer, and S.R. Das, "Ad Hoc On Demand Distance Vector (AODV) Routing," Internet Draft, draft-ietf-manet-aodv-10.txt, March 2002 (Work in Progress).

14. T. Camp, J. Boleng et V. Davies, "A survey of mobility models for ad hoc network research", Wireless communications mobile computing (WCMC): special issue on mobile ad hoc networking Research, trends and Application, vol, 2, p483-502, 2002. 